\title{
ON LOBULAR AND LOBAR PNEUMONIA IN INFANCY AND CHILDHOOD.
}

BY

OLIVE SOMERVILLE, M.B.,

Muirhead Scholar.

(From the Medical and Pathological Departments of the Royal

Hospital for Sick Children, Glasgow.)

Although much has been written and many statistical studies published on the relative frequency and age incidences of the different types of pneumonia in childhood, anything but unanimity prevails. This diversity of opinion is most marked regarding pneumonia as it occurs during infancy and early childhood, and is undoubtedly dependent on the different criteria which the individual workers have adopted for the differentiation of the lobar and lobular types of the disease. Some observers have based their classification on clinical grounds, believing that a definite diagnosis is possible from the history of the illness, the physical signs, the type of fever and degree of toxæmia present. This is a belief, however, which in our experience is not substantiated by the pathological findings. Others ${ }^{1}$ lay stress on the type of shadow revealed on the fluoroscope which they state is characteristic for each type of the disease. Others again have used a bacteriological basis of classification, while a small minority have employed the anatomical characters of the lesion as found at autopsy.

As in our opinion it is only on the basis of the anatomical nature of the lesion that any reliable decision on the matter can be reached, and as only relatively few of the previous studies of this question have been of this nature, we decided to investigate the subject from this point of view and the present communication is a record of the results obtained.

Material and Methods. The material investigated consisted of all the acute pneumonic conditions examined post-mortem in the Royal Hospital for Sick Children, Glasgow, from January 1925 to June 1926, and amounted in all to 65 cases. Pieces of tissue were taken from the various lobes of the lungs and were fixed in (1) ten per cent. formalin solution, (2) Zenker's solution, and (3) a saturated solution of bi-chloride of mercury. Paraffin sections, generally about a square inch in size, were stained with (1) Harris's hæmatoxylin and eosin; (2) Gallego's modification of Mallory's method ${ }^{2}$; (3) Weigert's fibrin $\operatorname{stain}^{3}$; (4) Iron hæmatoxylin; (5) Methylene blue and eosin.

Anatomical Features of Various Types of Pneumonia.

Anatomically the chief distinguishing features of each type may be summarized as follow. Lobar pneumonia spreads by direct continuity of ung tissue and leads to massive consolidation with a lobar distribution; the 
disease may spread more widely until it involves almost the whole lung, but it is not common to find two separate areas in the same lung. Bronchopneumonia, on the other hand, shows a patchy distribution, with lesions widespread throughout both lungs, though one lung may be much more affected than the other : an entire lobe is rarely consolidated.

The histological picture in lobar pneumonia is fairly constant. The chief feature is the abundant exudate of fibrin and polymorphs into the alveoli, septa, and the bronchioles. There is universal involvement of every portion of the lobe and of the different structures in the lobe. In spite of this intense inflammatory affection of the lung, there is, in the acute stage of the lobar type, very little cellular infiltration of the interstitial tissue in contrast to the marked interstitial changes in broncho-pneumonia. This has been pointed out by $\mathrm{McCallum}^{4}$ as a striking and distinctive feature in which the two forms differ.

The picture in broncho-pneumonia is more complex, but on section of the lung, the lobular distribution of the lesion can usually be made out, patches of dense leucocytic exudate being found in relation to the bronchioles and infundibular passages. The margins of the consolidated areas are ill-defined, irregular in outline, and separated by healthy, collapsed or emphysematous lung. The abundant fibrin formation, so constant in the lobar variety, does not occur. Some fibrin, however, is seen occasionally in the alveoli immediately surrounding the affected bronchioles, and this is said ${ }^{5}$ to befound more often in the influenzal than in any other form. The inflammation is largely catarrhal in nature with a few polymorphonuclear cells, though in some cases polymorphs are the predominating cellular elements. As previously noted, the interstitial tissue is always markedly affected, being infiltrated with large numbers of cells. ${ }^{5,6}$

Although in typical cases the histological features of the two forms are quite distinct, many intermediate forms, which partake of the characters of both, are recognized. In such cases the points to be relied upon for diagnosis are (1) the nature of the exudate, (2) the implication of the interstitium, and (3) the mode of spread as evidenced by a patchy cellular reaction.

Results.

From the study of the morbid anatomy and histology in conjunction with the clinical histories, which were employed to separate the primary from the secondary forms, the 65 cases have been classified as follows :

\section{BRONCHO-PNEUMONIA :}

(1) Primary broncho-pneumonia, in which the onset was either sudden or gradual in a previously healthy child: this group includes some cases with a clinical history indistinguishable from lobar pneumonia but in which lobular lesions were found on pathological examination.

(2) Secondary broncho-pneumonia, in which the pneumonia followed on some preceding disease, such as $(a)$ infectious fevers, disease of of respiratory passages (e.g., bronchitis), urinary tract, or nervous 
system; (b) chronic disease or malnutrition, e.g., marasmus, pyloric stenosis, syphilis, tuberculosis, congenital disease of the heart, tetany, rickets, and gastro-enteritis ; and $(c)$ following operations.

(3) Influenzal broncho-pneumonia.

\section{Lobar Pneumonia.}

The relative number of the different types and the age distribution from the findings in 65 cases are given in Table I.

\section{TABLE I.}

Showing Age Incidence of Cases Examined Histologically.

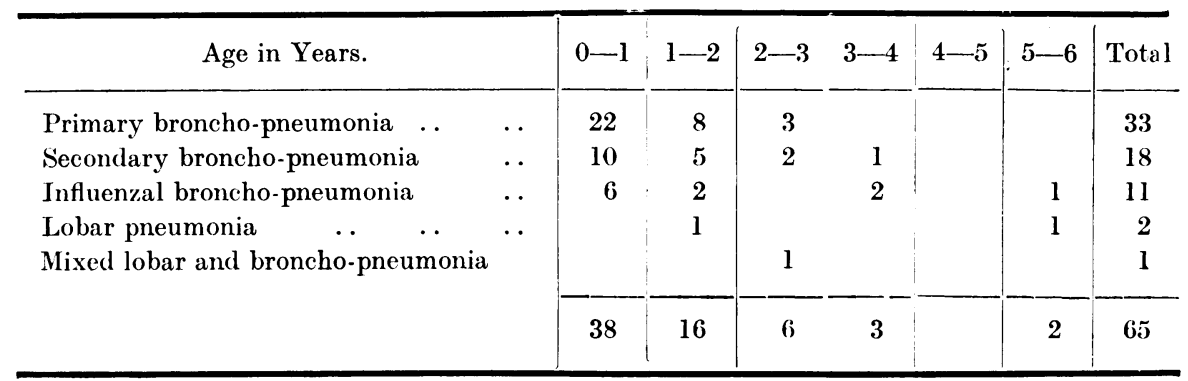

\section{(1) Primary Pneumonia.}

Under this heading primary broncho-pneumonia and lobar pneumonia will be discussed together, as most difficulty was found in the differentiation of these two forms. Thirty-six cases were examined, thirty-three of these being lobular in type, two lobar, and one mixed. Excepting one of the cases of lobar pneumonia, all occurred in children below threeyears of age. Of the thirtythree lobular forms, seventeen were diffuse and in three cases closely simulated lobar pneumonia; the remaining sixteen showed typical lobular lesions.

On microscopical examination of all the cases of broncho-pneumonia, the lobular distribution could be made out at some part. In many cases by the aggregation of foci the consolidation had spread through almost an entire lobe, giving the appearance of hepatization, but the microscopical features were more of the lobular type, areas of emphysema being intermingled with densely cellular areas, and typical lobular lesions were found in other lobes.

The commonest lesions were those of a pneumonia of about two weeks' duration, and the conditions found in such circumstances will first be described. The bronchi showed desquamation of epithelium varying in degree in different parts of the same section, but seldom as severe as in the smaller bronchioles. Their lumina were plugged with a cellular exudate of polymorphs and desquamated epithelial cells, the latter often in plaques, with a few red blood cells and small round cells. The bronchial walls were congested and often 
infiltrated with numbers of small round cells. According to Gaskell, ${ }^{7}$ this lymphocytic peribronchial reaction varies directly with the virulence of the infecting organism. There was less desquamation of the lining epithelium of the bronchi than of the bronchioles, some parts generally remaining covered with normal epithelium. Sometimes, in the smaller bronchi, only a thin layer of polymorphonuclear and epithelial cells was to be seen on the top of the basement membrane lining the lumen. In a few of the diffuse types, fibrin was found extending through the walls into the air vesicles immediately surrounding some of the more severely affected bronchi.

The desquamation of the lining epithelium of the bronchioles in the consolidated areas was always severe, the walls being either partially or completely denuded. A densely cellular exudate of polymorphs and desquamated epithelial cells completely filled the lumen, the polymorphonuclear exudate extending directly into the alveoli through the infundibular passages. Definite suppurative change was found in 35 per cent. of the cases : in these the basement membrane showed a break in continuity and polymorphonuclear cells were invading the surrounding lung tissue. In more than half of the cases lymphocytic reaction was present round the bronchioles : this was very marked in nine cases in which several aggregations of small, round cells were seen in the walls. In the lobular forms no fibrin was present, but some of the diffuse types showed a little extending through the bronchiole walls into the adjacent alveoli.

The air vesicles round the affected bronchi and bronchioles were consolidated in an irregular, patchy manner. In the lobular forms, the areas of consolidation were small, and in the centre of each, one or more plugged bronchus or bronchiole could be distinguished, and the alveoli surrounding the patch were emphysematous. The diffuse types, however, showed widespread consolidation, and in some sections no unaffected alveoli could be seen, as the patcheshad completely merged into one another. The cellular exudate was in twenty-one cases mainly polymor phonuclear, but in the remaining twelve cases small round cells and catarrhal cells predominated. In several cases it was noted that the exudate in the bronchioles was wholly polymorphonuclear, and the cell reaction in the alveoli consisted of mononuclear cells.

The density of the consolidation always varied considerably in different parts : some alveoli were packed tightly with a mass of polymorphonuclear ; some contained mucoid and granular material with only a few cells in various stages of disintegration ; and others contained chiefly large mononuclear cells. The cellular exudate was always more abundant in the air vesicles immediately surrounding the bronchial passages than in those at the margin of the patch ; and sometimes definite zones of inflammation could be made out resembling successive stages of lobar pneumonia, these being from within outwards (1) grey hepatization, (2) early red hepatization, and (3) primary catarrhal stages. ${ }^{7}$ Areas of œdema with hæmorrhage were occasionally seen, but these were both more common in the influenzal forms. Interspersed among the affected areas were emphysematous alveoli or single distended air vesicles in which no exudate 
was present. Small areas of collapse were often found round the terminal bronchioles, at the margin of the consolidation, or, as described by Armstrong and Gaskell, ${ }^{8}$ under the pleural surface.

The interstitial tissue was invariably affected to a greater or lower degree, depending on the extent of the lesion. The alveolar walls were swollen and infiltrated with cells, sometimes thereby encroaching on the alveolar spaces to a marked degree. In the more advanced stages where suppurative change had taken place the walls of the air vesicles were indistinguishable, and masses of polymorphonuclear, with a broken down bronchiole in the centre, were all that remained. In several cases the interstitial tissue between the lobules, and in the neighbourhood of the bronchi and vessels, was increased in size on account of infiltration with polymorphonuclear and deposit of fibrin.

A slight fibrinous layer was found on the pleura where the consolidation had reached the surface of the lung, but extensive pleurisy such as is usually seen in lobar pneumonia was never present. Pneumococci were invariably found in the pus from the finer bronchi, seldom in pure culture, but generally mixed with streptococci, pneumobacilli, and occasionally also with staphylococci.

The above description applies to all types of primary broncho-pneumonia encountered. In sixteen cases the lobular distribution was easily recognized both macroscopically and microscopically, and the clinical history was also typical of that form of the disease. In the eighteen remaining cases of lobular pneumonia and in one of the lobar cases, more difficulty was found in differentiation, and prolonged histological examination was necessary before they could be finally classified. Fourteen of these showed the characteristics of a lobular lesion which, by fusion of adjacent foci of consolidation, involved the greater part of one lobe, and so simulated lobar pneumonia on naked-eye examination. Histologically, however, cellular reaction was found to be most intense round the bronchi, the exudate being irregular in density and nature, parts of the same lung showing a polymorphonuclear exudate, whilst in other parts large mononuclear cells predominated. Fibrin was scanty, being found in only six cases and always confined to the air vesicles immediately surrounding the bronchioles.

The remaining five cases showed features of both lobar and lobular types and the following summary of each case will demonstrate the difficulties encountered in arriving at a satisfactory conclusion regarding these forms.

Case 1. Boy, aged 1 year, 10 months. He was drowsy and off food for about one week before the onset of cough and dyspnœa. Two days later he had a rigor. His symptoms in. creased in severity until, three days later, he was admitted to hospital where he died twenty-six days afterwards. During this time, the physical signs of pneumonia were limited to the base of the left lung. The temperature was irregular, between $99^{\circ}$ and $103^{\circ} \mathrm{F}$., and the pulse and respiration rates were correspondingly high. The leucocytes numbered 28,000 per c. $\mathrm{mm}$.

At the autopsy, there was found a diffuse consolidation of the entire left lower lobe, apparently of lobar type and in the stage of red hepatization, the other lobes being unaffected. Fibrinous pleurisy and early pneumococcal pericarditis were also present. On histological 
examination the consolidation was found to be loose and fairly uniform, cells were polymorphonuclear and mononuclear, but the reaction was most intense around the bronchioles, fibrin was absent, and there were occasional unaffected alveoli. The pneumococcus alone was found on culture of the pus from the smaller bronchi.

In this case the symptomatology was atypical, and the physical signs and naked-eye postmortem findings were those of lobar pneumonia. It was only on histological examination that it was possible to make out the lobular nature of the lesion.

Recovery would probably have taken place if the additional burden of pericardial infection had not supervened in a child already worn out by a long continued fever, and the case would have been classified as one of lobar pneumonia.

Case 2. Girl, aged 1 year, 2 months. Since bronchitis at eight months she had never been well. Ten days before admission, cough and heavy breathing developed, and gradually increased in severity. On admission the child was very ill with physical signs of pneumonia of the entire right lung and the left apex; the condition remained unchanged till death eight. days later. The temperature was persistently high, and the pulse and respiration rates correspondingly increased. The leucocytes were 97,600 per c. mm. on admission.

At the autopsy, a diffuse lesion was found involving almost the entire right lung with tho exception, in each of the lobes, of a small portion of the anterior edge which was the seat of acute vesicular emphysema. The line of demarcation between the consolidated area, which resembled the grey stage of a lobar pneumonia, and the emphysematous area was very irregular, the one passing imperceptibly into the other. Fibrinous pleurisy was present over the surface of the right lung. The left upper lobe was the seat of typical lobular lesions. Histologically, the most notable features were the abundant fibrinous exudate extending throughout the alveoli, and the marked peribronchial polymorphonuclear reaction. The interstitial tissue was affected to a considerable extent, being infiltrated with fibrin and large numbers of polymorphonuclear cells.

Clinically this case showed many of the features of lobar pneumonia in the severity of the general symptoms and the maintenance of high fever throughout its course, but the mode of onset and the physical signs were more of the broncho-pneumonic type. The lesion in the right lung resembled in some respects a lobar pneumonia on naked-eye examination, but the histological changes, though somewhat atypical on account of the large amount of fibrinous exudate, were more of the nature of a lobular form of infection, and the fact that a definite bronchopneumonic consolidation was present in the other lung gave additional support to this conclusion.

Case 3. Girl, aged $2 \frac{1}{2}$ years. The symptomatology was that of a pneumonia of fairly acute onset and course ; death taking place after an illness lasting three weeks. The following lesions were found at the post-mortem examination : (1) consolidation of the entire right lower lobe, with collapse of right upper and middle lobes ; (2) large right-sided empyema ; (3) slight pericarditis ; (4) bilateral otitis media. On histological examination of portions of the affected lung, a mixed type of lesion, in many respects resembling lobar pneumonia, was found. There was diffuse consolidation of the air spaces with much fibrinous exudate, but cells, though numerous in the bronchi, were scanty in the air vesicles and were mainly mononuclear in type. The desquamation of the lining epithelium of the bronchi and bronchioles was almost complete, their lumina being plugged with dense collections of polymorphonuclear cells with marked degenerative changes in their nuclei. The exudate in the bronchioles was observed to be directly continuous with that in the air vesicles. Peribronchial lymphocytic reaction was very marked. No areas of emphysema, collapse, œdema, or hæmorrhage were present in the sections examined, but there was much suppurative change of a characteristic lobular type.

In this case an entire lobe was affected. The lesion was associated with empyema, a fact thought by Still $^{y}$ to be almost pathognomonic of co-existing lobar pneumonia, and the exudate was largely fibrinous in nature. All these points were in favour of a lobar lesion, yet the 
histological features, especially the secondary suppurative change, were more characteristic of broncho-pneumonia. Such an intense affection of the air passages and so marked a peribronchial reaction are never found in a lobar pneumonia.

Case 4. Boy, aged 2 years. The illness began with diarrhœa and was followed two weeks afterwards by cough, fever, and rapid breathing. He was admitted to hospital one week subsequently when the symptoms had increased in severity, with the physical signs of a right-sided pneumonia. Death occurred three weeks later.

At the autopsy, a diffuse, uniform consolidation, granular in appearance and suggesting red hepatization, was found to involve the entire right upper lobe, although the middle and lower lobes showed typical lobular lesions. There was fibrinous pleurisy on the surface of the right lung. The left lung was normal in appearance. Sections of the right upper lobe showed in the air vesicles a diffuse exudate of fibrin with polymorphonuclear and small round cells. The desquamation of the epithelium of the bronchi and bronchioles was not severe, and all were affected more or less to the same degree. Peribronchial lymphocytic reaction was slight, the interstitium was practically unaffected, and there were few catarrhal cells and no areas of emphysema or collapse. The pneumococcus alone was found in the pus from the smaller bronchi. The lesions in the other lobes were typically lobular in type.

The lesion in the right upper lobe in this case was characteristically lobar in distribution, in its post-mortem appearances, and its histology, though the symptomatology was not typical of this disease. The fact that lobular lesions were present in other lobes may account for the atypical course of the illness.

Case 5. This case, though lobar in type, showed many atypical features and will be described briefly to compare with the above four cases.

Boy, aged 1 year. He was admitted to hospital moribund, with the history of fever and heavy breathing of three weeks' duration. There was severe anæmia: hæmoglobin, 10 per cent.; red cells, 2,500,000 ; and leucocytes, 2,000 per c. mm. He died a few hours after admission.

At the autopsy extensive consolidation, greyish and uniform in distribution, was found involving the entire right upper lobe and the lateral part of the middle lobe, the rest of the lungs being unaffected. Associated with this were left acute otitis media, and longitudinal sinus thrombosis with meningeal hæmorrhage. On histological examination of portions of the lung involved, a very diffuse, fairly uniform consolidation was found, and an exudate composed of polymorphonuclear cells and much fibrin. In some parts the exudate had retracted from the alveolar walls. The desquamation in the bronchioles was only moderately severe, but there was marked peribronchial lymphocytic reaction. Many large mononuclear cells were present in some parts. There was slight cellular infiltration of the interstitial tissue and there were no areas of emphysema, collapse, or hæmorrhage.

This case, though showing a few of the features of broncho-pneumonia, namely, the peribronchial lymphocytic reaction, was certainly more of a lobar type of consolidation as the fibrinous exudate was uniform in distribution. The air passages, too, were less involved than would be expected in such a severe broncho-pneumonic infection, and the interstitium was practically unchanged. In addition, it is very unusual to find the other lobes of the lungs unaffected in a severe confluent form of broncho-pneumonia. The conclusion was therefore drawn that this was a lobar pneumonia.

Both clinical and post-mortem findings did not correspond to the classical types in all five cases. In each case one lobe at least was consolidated in its entirety, thus simulating the stage of red or grey hepatization of a lobar pneumonia, and in only two cases was there any lobular consolidation in other parts. The only naked-eye characteristics pointing to the lobular nature of 
the lesion were the irregular margins of consolidation in Case II and the type of suppuration in Case III.

Even the histological findings were by no means typical. Four cases showed such an abundant fibrinous exudate as is rarely found in acute bronchopneumonia, and no areas of emphysema or collapse were found in the consolidated area. The first three cases were distinguished as lobular in type, the significant features being, first, the evidence of bronchial spread from the marked affection of the air passages and intense peribronchial reaction, and secondly, the affection of the interstitium. The fourth case showed lobar and lobular lesions, and the fifth was lobar in nature, though atypical in some of its features.

These five cases represent very different forms of pneumococcal infection and illustrate some of the difficulties encountered in reaching a correct classification even after careful histological examination. A feature common to all was the occurrence of the disease in the second or third year of life. In the cases occurring during the first year and after the third year there was never any doubt regarding the nature of the lesion; it was only those cases occurring during the intervening period (second and third years) which presented difficulties in classification. It might be inferred from this fact that a transition takes place at this time, the pneumonia gradually losing its lobular character and approaching the lobar form of the adult. In support of this view may be quoted the opinion of M. Valleix ${ }^{10}$, who, as early as 1850 , described similar differences according to age. He divided the cases into three periods: (1) up to two years, (2) two to six years, and (3) six to fifteen years, and regarded the second period as one of transition from the invariable lobular form of the first period to the lobar form of the third ; the fatality of the disease was found to diminish correspondingly.

To compare with these mixed forms, details of the only case of typical lobar pneumonia in the whole series are appended.

Case 6. Boy, aged 5 years. The history was that of an acute illness of three weeks' duration, with marked general symptoms, high fever, and definite physical signs of consolidation of the right lung.

At post-mortem examination, complete consolidation of the right lung was found with a localized empyema, slight pericarditis, bilateral acute otitis media, and pneumococcal meningitis: the left lung was unaffected. Sections of the right lung showed extensive fibrinous consolidation of uniform density. The cells were chiefly polymorphonuclear showing much fragmentation of nuclei. There was present fairly severe catarrh of the bronchioles, and to a lesser degree of the broachi, with marked peribronchial infiltration by small, round cells; but there was not much variation in the degree of change, and no free alveoli. There was some early diffuse suppurative change. The interstitium was practically unaffected; there were no areas of catarrhal inflammation and no collapse. The pneumococcus, staphylococcus, and pneumobacillus were all found in pus from the fine bronchi; and the pneumococcus was found in the pus from the surface of the brain, and from both middle ears.

The great differences between this case and those previously cited were the uniformity of the lesion, the slighter degree of affection of the bronchioles 
and interstitium, and the absence of catarrhal inflammation. In this case, also, the clinical and post-mortem features agreed, and were typical of the lobar form of the disease.

\section{(2). Secondary Broncho-Pneumonia.}

In the examples of secondary pneumonia, the pathological picture was more constant and conformed fairly closely to the typical lobular form already described. Seventeen of the total eighteen cases were of this type. The lesions were of a patchy distribution, affecting both lungs in more than half of the cases, and often being at a more advanced stage in the posterior parts of the lower lobes. Microscopically, the chief features were the lobular distribution of the consolidation, with marked affection of the bronchi and bronchioles, and areas of emphysema and collapse. Fibrin was usually absent, and œdema and hæmorrhage frequently occurred. In eleven cases the cellular exudate consisted of polymorphonuclears and mononuclears, though polymorphonuclears were more abundant in the bronchi; in the other seven cases polymorphonuclears predominated throughout the consolidated area. Peribronchial lymphocytic reaction was very marked in one-half of the cases ; clumps of small, round cells being present in the walls of the bronchi and vessels. Catarrhal cells were found in large numbers in all cases. Suppuration was the exception. Pleurisy only occurred in two of the cases when there was a scanty deposit of fibrin on the pleural surface.

In only one case did the infection show any tendency to spread through an entire lobe in a diffuse manner. Here the condition was secondary to a sub-acute nephritis.

Case 7. Girl, aged $3 \frac{3}{4}$ years. The history was of generalized cedema of two week's duration with the development of cough, fever, and rapid breathing one week before death. The lungs showed a diffuse consolidation of all the lobes with much hypostatic congestion, œdema, and some hypostatic pneumonia. There was a little clear fluid in both pleural sacs. A definite sub-acute nephritis was present.

Sections of portions of the lung showed a diffuse fibrinous consolidation, loose and irregular in density, with intense peribronchial lymphocytic reaction, and practically complete desquamation of the epithelium of the bronchioles. A dense polymorphonuclear exudate, continuous with that in the air vesicles, was present in the bronchioles; and in places the walls of the air vesicles showed breaking down on account of suppurative change. In some regions there were numbers of round and large mononuclear cells. No areas of collapse were seen, and none of hæmorrhage.

Though this case showed a lobar distribution, the histological features were those of a lobular pneumonia with aggregation of adjacent foci of consolidation, œedema being present in the intervening areas, The diagnosis by microscopical examination presented very little difficulty.

\section{(3). Influenzal Broncho-Pneumonia.}

The lesions most often found in the influenzal forms of pneumonia were similar to those of a diffuse primary broncho-pneumonia of a very severe type. Eleven cases were examined and in all of these a bacillus corresponding to Pfeiffer's influenzal bacillus was isolated from the pus in the finer bronchioles ; 
in the larger bronchi, pneumococci and streptococci were also present. Eight of the cases occurred in the first two years of life, two in the fourth year, and one in the sixth year (see Table I). The duration of the illness was in all cases less than three weeks, in six being under one week. Extensive lesions were found in both lungs; in seven cases every lobe was affected, and in the remaining four cases foci were present in both lower lobes. In six of the cases on naked-eye examination there was diffuse consolidation of the lower portion of a lower lobe simulating red hepatization, but the upper margin was irregular and showed a less advanced stage of consolidation. Emphysema was always marked in the free parts, much more so than in any other form of pneumonia. Microscopically, the consolidation was invariably bronchial in distribution and cellular in nature; fibrin, when present, was confined to the air vesicles in immediate relation to the bronchi. Bronchitis and bronchiolitis were severe, and in most cases suppuration had occurred. In the types which showed lobular consolidation on naked-eye examination, and in the less advanced parts of the diffuse forms, both polymorphonuclear and mononuclear cells were present, fibrin was scanty, and the consolidated areas, which varied in size, always surrounded a bronchiole and were separated from one another by areas of compensatory emphysema. In the diffuse forms, the consolidation still retained its lobular character ; both mononuclears and polymorphonuclears were present but more numerous in the suppurative areas, in the lumina of the bronchi and bronchioles. There were many areas of hæmorrhage and collapse. Peribronchial lymphocytic reaction was only slight. Large mononuclear cells were present in all cases but were scanty. Pleurisy was only present in three cases.

\section{Conclusions.}

From this study of the histology of pneumonia it is evident that broncho. pneumonia is the typical lesion of infancy and early childhood, and is the invariable type in the secondary and influenzal forms. It may be concluded, also, that lobar pneumonia, although it does occur, is the exception during the first three years of life, and never takes the typical form of the same disease as found in the adult. During this period, however, certain mixed forms with many of the characteristic features of lobar pneumonia do occur, and can only be distinguished by a careful histological examination. To this end, the simplest and most conclusive method would be by making histological sections of the entire lung, for this would show the mode of spread of the inflammatory process and the extent of the lesion. In the present investigation, the means available did not allow of large sections being made. These mixed forms occur most frequently during the second and third years of life. After the age of three years, the disease is more often lobar in type though it would appear from the post-mortem records that this lesion is occasionally associated with broncho-pneumonia. If the latter does occur alone after three years of age, it is usually either secondary or influenzal ; in the few cases in which it is primary, it occurs in an atypical form.

It is difficult to find an explanation of this variation in the character of the lesions in the earlier years of life. Gaskell, in his experimental work on 
rabbits, found great difficulty in producing lobar lesions and suggests that the size of the lung may be of some importance, the larger lung of the adult allowing time for concentration of the inflammatory reaction and therefore being more favourable for keeping the infection within the lobe. It has also been suggested that lobar pneumonia is always a pure pneumococcal infection, whilst in broncho-pneumonia a mixed infection is the rule, but this is not invariably so. In some cases of primary broncho-pneumonia, the pneumococcus alone is found, and on the other hand, a few cases of lobar pneumonia show a mixed bacterial flora.

Whatever the explanation, it would seem quite definite that the cause of the variation in the type of reaction is some inherent tendency in the child. This may be dependent either on some peculiarity of the lung itself or in consequence of some subtle serological or metabolic characteristic associated with the period of life. We are quite familiar with the varying susceptibility of different organs to a specific organism, e.g. the meningococcus; with the varying susceptibility at different age periods of any individual organ to a specific organism, e.g. rheumatic infection; with the varying type of reaction at different ages to a specific organism, e.g. tubercle bacillus; and with the varying susceptibility of an organ to an infection which occurs under special or unusual circumstances. Of the last phenomenon the immunity of the cerebrum to the Treponema pallidum in a person subject to malaria may be cited.

It thus seems possible that some subtle metabolic characteristic may bring about a special pulmonary reaction in response to infection with the pneumococcus. In this way there could be explained the different reactions in the infant and adult and also the mixed type of reaction which occurs during the stage of transition from the characters of the infant to those of the adult.

\section{SUMMARY.}

1. Sixty-five cases of pneumonia were examined by histological methods and a bacteriological examination was made in some of these.

2. Only one case of typical lobar pneumonia was found, this being in a boy of five years, but two atypical lobar forms were found in children of one and two years and in one of these there was, in addition, a lobular lesion in the other lung.

3. Of thirty-five cases of acute primary pneumonia examined, sixteen showed typical lobular lesions, fourteen were diffuse with a lobar distribution in one lobe, but easily differentiated on microscopical examination ; the remaining five were mixed types with features of both lobar and lobular forms and these occurred in the second and third years of life.

4. In the eighteen secondary cases, the lesions were typically lobular in distribution and in their histological features.

5. The influenzal cases showed characteristics of an intensely virulent infection. The lungs were extensively involved, six of the eleven cases showing almost a lobar distribution, but the consolidation was always lobular in nature. 
6. Pure pneumococcal infections occurred in both lobar pneumonia and primary broncho-pneumonia, but in many cases, the pneumobacillus, streptococcus and staphylococcus were also found, and in secondary and influenzal forms a mixed infection was invariable.

\section{REFERENCES.}

1. Armand-Delille, P. F., Brit. Med. J., Lond., 1927, ii, 588.

2. Med. Sc., Abs. \& Rev., Oxf., 1925, XI, 43.

3. Green's, Pathology, Lond., 1923.

4. MacCallum, W. G., Textbook of Path., Lond., 1923.

5. Muir, R., Textbook of Path., Lond., 1924.

6. Wollstein, M., \& Goldbloom, A., Am. J. Dis. Child., Chic., 1919, XVII, 165.

7. Gaskell, J. F., J. Path. \& Bact., Edin., 1925, XXVIII, 427.

8. Armstrong, R. R., \& Gaskell, J. F., Ibid., 1921, XXIV, 369.

9. Still, G. F., Com. Dis. \& Disorders of Child., Lond., 1924.

10. Valleix, M., Am. J. Med. Sc., Philad., 1850, XIX, 211. 